\title{
Classifying online handwriting characters under cosine representation
}

\author{
Bui T.D. \\ College of Technology, Vietnam National University, Hanoi
}

\begin{abstract}
The natural way of handwriting to enter data into computer is still preferable in many tasks. However, handwriting character recognition is not a trivial task for computer. Based on the presentation of the input, handwriting recognition can be divided into two classes: offline and online. The main advantage of online handwritten data over offline data is the availability of stroke segmentation and order of writing. Utilizing this information rather than static image only can obtain higher recognition rate [11]. In this paper, we extend the method proposed in [13] to represent multiple strokes of a character together in a single set of features using cosine transformation. Using this representation, we have developed an online writerindependent character recognition system with MultiLayer Peiveptron (MLP) classifiers, one classifier for each single character. We have tested our system on Section la (isolated digits) of the Unipen data set [7] and have obtained very competitive results. (c) 2007 IEEE.

Index Keywords: Character recognition; Classification (of information); Classifiers; Feedforward neural networks; Information technology; Linguistics; Technology; Character recognition system; Data sets; Handwriting recognition; International conferences; Language processing; Multi layering; Off-line data; Online handwriting; Online writer; Recognition rates; Static imaging; Web information; Learning systems
\end{abstract}

Year: 2007

Source title: Proceedings - ALPIT 2007 6th International Conference on Advanced Language Processing and Web Information Technology

Art. No.: 4460641

Page : 206-211

Cited by: 1

Link: Scorpus Link

Correspondence Address: Bui, T. D.; College of Technology, Vietnam National University, Hanoi; email: duybt@vnu.edu.vn

Sponsors: Korea Information Processing Society;Luoyang University of Foreign Language;KiSTi;NEOPAD;SoftonNet

Conference name: 6th International Conference on Advanced Language Processing and Web Information Technology, ALPIT 2007

Conference date: 22 August 2007 through 24 August 2007

Conference location: Luoyang, Henan

Conference code: 73055

ISBN: 0769529305; 9780769529301

DOI: 10.1109/ALPIT.2007.72

Language of Original Document: English

Abbreviated Source Title: Proceedings - ALPIT 2007 6th International Conference on Advanced Language 


\section{Processing and Web Information Technology}

\section{Document Type: Conference Paper}

Source: Scopus

Authors with affiliations:

- Bui, T.D., College of Technology, Vietnam National University, Hanoi

References:

- Ahmed, N., Natarajan, T., Rao, K.R., Discrete cosine transform (1974) IEEE Trans. Computers, C-23 (1). , January

- Becker, P.W., Nielsen, K.A., Pattern recognition using dynamic pictorial information. IEEE Transaction in System, Man (1972) Cybernetics, SMC-2. , July

- Bellegarda, J.R., Nahamoo, D., Nathan, K.S., Automatic handwriting recognition using both static and dynamic parameters. (1996), United States Patent number 5,491,758, FebruaryConnell, S., Jain, A., Template-based online character recognition (2001) Pattern Recognition, 34 (1). , January

- Connell, S.D., Jain, A.K., Learning prototypes for on-line handwritten digits (1998) Proceedings of the 14th International Conference on Pattern Recognition, 1

- Gagne, C., Parizeau, M., Genetic engineering of hierarchical fuzzy regional representations for handwritten character recognition (2006) International Journal of Document Analysis and Recognition, 8 (4), pp. 223-231. , September

- Guyon, I., Schomaker, L., Plamondon, R., Liberman, M., Janet, S., Unipen project of on-line data exchange and recognizer benchmarks (1994) Proceedings of the 14th International Conference on Pattern Recognition (ICPR)

- Hagan, M., Demuth, H., Beale, M., (1995) Neural Network Design, , PWS Publishing Company

- Hebert, J.F., Parizeau, M., Ghazzali, N., A new fuzzy geometric representation for on-line isolated character recognition (1998) Proceedings of the 14th International Conference on Pattern Recognition

- Li, X., Parizeau, M., Plamondon, R., Segmentation and reconstruction of on-line handwritten scripts (1998) Pattern Recognition, $31(6)$

- Mandler, E., Oed, R., Doster, W., Experiments in online script recognition (1985) Proceedings of 4th Scandinvian Conference in Image Analysis, , June

- Parizeau, M., Lemieux, A., Gagne, C., Character recognition experiments using unipen data (2001) Proceedings of 6th International Conference on Document Analysis and Recognition (ICDAR)

- Polyakov, V.G., Ryleev, M.A., Method and apparatus for representing image data using polynomial approximation method and iterative transformation-reparametrization technique. (1995), United States Patent number 5,473,742, DecemberSuen, C., (1986) Computational Studies of the Most Frequent Chinese Words and Sounds, , Word Scientific, Singapore

- Tappert, C.C., Suen, C.Y., Wakahara, T., The state of the art in on-line handwriting recognition (1990) IEEE Transaction on Pattern Analysis and Machine Intelligence, 12 (8)

- Teredesai, A., Govindaraju, V., Issues in evolving gp based classifiers for a pattern recognition task (2004) Congress for Evolutionary Computation 04 (CEC 04)

- Teredesai, A., Ratzlaf, E., Subrahmonia, J., Govindaraju, V., On-line digit recognition using off-line features (2002) Proceedings of Indian Conference on Computer Vision, Graphics and Image Processing 